\title{
A Survey and Theoretical View on Compressive Sensing and Reconstruction
}

\author{
Santosh S. Bujari \\ SKSVMACET/ECE, Laxmeshwar, 582116, India \\ Email: santoshbujari@gmail.com \\ Saroja V.Siddamal \\ BVBCET/ECE, Hubli, 580021, India \\ Email: sarojavs@bvb.edu
}

\begin{abstract}
Most of the current embedded systems operate on digital domain even though input and output is analog in nature. All these devices contain ADC (Analog to Digital converter) to convert the analog signal in to digital domain which is used for processing as per the application. Images, videos and other data can be exactly recovered from a set of uniformly spaced samples taken at the Nyquist rate. Due to the recent technology signal bandwidth is becoming wider and wider. To meet the higher demand, signal acquisition system need to be improved. Traditional Nyquist rate which is used in signal acquisition suggests taking more numbers of samples to increase the bandwidth but while reconstruction most of the samples are not used. If samples are as per Nyquist rate then, this increases the complexity of encoder, storage of samples and signal processing. To avoid this new concept Compressive Sensing is used as an alternative for traditional sampling theory. This paper presents a survey and simplified theoretical view on compressive sensing and reconstruction and proposed work is introduced.
\end{abstract}

Index Terms - CS, Sparse Matrix, Sparsity, RIP, NSP.

\section{INTRODUCTION}

Due to Nyquist rate much of the signal processing has moved from the analog to the digital domain. As a result of this success the amount of data generated by sensing systems has grown from a smaller number to a larger number. Unfortunately in many important and emerging applications the resulting Nyquist rate is so high that we end up with far too many samples and it may be too costly or even physically impossible to build devices capable of acquiring samples at that necessary rate. After 20th century onwards amount of data generated grows in super exponential form which means data growth was about 30 to $40 \%$ per year. This amount of data generated exceeds the total storage available in the word. This is called DATA DELUGE problem. Even though storage capacity is increased but transmission rate or channel rate is not increased up to the mark. Rate of date generation is much larger than rate of increase in transmission rate. Compressive Sensing concept [2], [3], [5] and [11] depicts that one can recover certain signals from less number of samples than Nyquist rate. Compressive Sensing is asymmetric which means it consist of dumb encoder and smart decoder. But it is a stable system [9]. This paper contains seven sections. First section includes introduction and data deluge problem, where as second section explains few fundamental facts such as Sparsity and Normed vector spaces. Third section contains relationship between CS theory and sampling theory. Fourth section contains theory related to design of sensitive matrix. Fifth section explains reconstruction algorithm in the form of graphical methods. Last two sections contain proposed work, literature survey and conclusion with future work.

\section{FUNDAMENTAL FACTS OF CS}

\section{A. Sparsity Property}

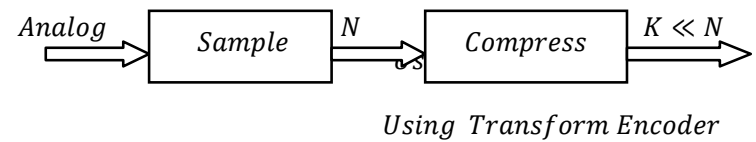

Fig.1. General Compression.

General compression as shown in Fig.1 when analog object is uniformly sampled then it generates ${ }^{\prime} \mathrm{N}^{\prime}$ number of samples. These samplers are large in number and reduced using Transform coders. These coders compress the signal in to smaller numbers ' $\mathrm{K}$ ' by converting from one domain (basis) in to other domain. Suppose the information is in image domain of dimension ' $N$ ' then it is converted into Wavelet or DCT (Discrete Cosine Transform) domain of dimension'N'. After analysing 'N' dimensional wavelet or DCT samples it is found that most of the coefficients are very small and some coefficients (K) are large. Small coefficients are truncated and remaining coefficients are used for further processing. Finally signal is compressed from dimension ' $N$ ' to dimension ' $\mathrm{K}$ ' which contains all the intrinsic information about the object. This concept was almost used in JPEG coder. This property is called sparsity and representation of analog object into few numbers like wavelet or DCT domain is called sparse representation [12]. All kinds of 
signals are naturally sparse in nature either in sampled domain or in other domain. Most of the practical signal contains sparsity nature. Even noise itself exhibits sparsity property. Sparse vector contains all zeros and few (K) nonzero entries. This property is represented mathematically using matrix called sparse matrix. This is defined as "any matrix with enough zeroes values that it pays to take advantage of them". In compression these values are truncated and thus signal dimension is reduced. In time domain sparse signal is a simple vector of length ' $N$ ' in which only ' $\mathrm{K}$ ' elements has nonzero value which is called as Canonical Sparse. When these elements are sorted they will obey the power law decay. Sparse signal always live in high dimensional or infinite dimensional spaces which are aligned with coordinate axis. For example if sparse vector contains three elements and one of them is zero means zero can be located any location of the vector. It seems to be very simple but these elements live in three separate two dimensional spaces which are aligned with coordinate axis as shown in Fig. 2 and are orthogonal to each other.

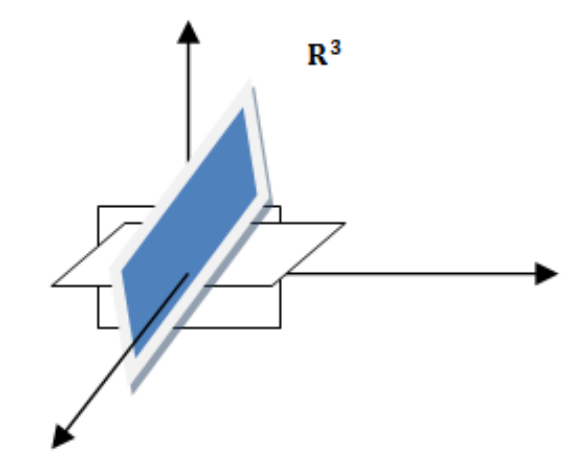

Fig.2. Three dimensional subspace- $\mathrm{R}^{3}(\mathrm{~N}=3, \mathrm{~K}=1)$

A set of sparse signal is point in million dimensional spaces that live along some (ex: 10000) sub dimensional space as shown in Fig. 3 which are aligned with coordinate axis.

\section{$R^{N}$}

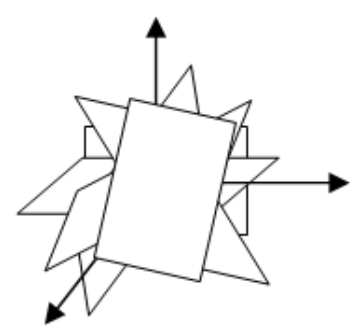

Fig.3. Million Dimensional Space

Compressible signal is a set of sorted elements of sparse vector of length ' $\mathrm{K}$ '. Each element of a vector is either wavelet or DCT coefficients $(\mathrm{Ci})$. Sort the vector coefficient according to its magnitude then it follows the power law decay property. A set $\{\varnothing \mathrm{i}\}$ where $\mathrm{i}=(1,2,3 \ldots \ldots \mathrm{n})$ is wavelet or DCT basis for $\mathrm{R}^{\mathrm{N}}$ are linearly independent. This implies that each vector in the space has a unique representation in the form of linear combination which is depicted in equation (1). $\mathrm{X} \in \mathrm{R}^{\mathrm{N}}$, there exist coefficients $\{\mathrm{Ci}\}$ where $i=(1,2,3 \ldots \ldots \mathrm{n})$ such that

$$
\mathrm{x}=\sum_{\mathrm{i}=1}^{\mathrm{n}} \mathrm{Ci} \varnothing \mathrm{i}
$$

It means signal is sparse in some other domain (Øi) with value $\mathrm{Ci}$ then time domain signal can be expressed using above formula which is called as dual basis formula. Let Fì denote the $\mathrm{n} \times \mathrm{n}$ matrix with column given by $\emptyset_{i}$ and $F_{i}$ denotes entries then complete signal $\mathrm{F}$ can be completely represented by equation (2).

$$
\mathrm{x}=\Phi \mathrm{c}
$$

Two wavelet or DCT bases are said to orthonormal if they satisfy following condition as shown in equation (3).

$$
\left(\emptyset \mathrm{i}, \varnothing_{j}\right)= \begin{cases}1, & i=j \\ 0, & i \neq j\end{cases}
$$

An orthonormal basis has the advantage that the coefficient $\mathrm{F}$ can be easily calculated from equation (4).

$$
\mathrm{c}=\Phi^{\mathrm{T}} \mathrm{X}
$$

By using the sparsity concept if we express only those sets which are linearly dependent then those basis are called as frame. Naturally dimension will be reduced from $\mathrm{F}$ to $\mathrm{F}$ and matrix dimensions will be reduced $\mathrm{d} \times \mathrm{n}$ such that all sparse $x \in \mathrm{R}^{\mathrm{d}}$. This concept is extended in CS for signal reconstruction.

\section{B. Normed Vector Space}

Every system can be modeled in a linear fashion. Each system process the signals to produce proper results, therefore signals should be in linear form. Linear property indicates that when we add signal it should produce new kind of signal. In vector space using length, angel and distance of each signal is used to estimate the proper value. Mathematically signals are viewed as vectors in an ndimensional Euclidian space, denoted by $\mathrm{R}^{\mathrm{N}}$. Using Lp norms these signals are analyzed. In mathematics, the Lp spaces are function spaces defined using a natural generalization of the form $\mathrm{p}$-norm for finite-dimensional vector spaces. These Lp norms are defined as shown in equation 5 .

$$
\|x\|_{p}=\left\{\begin{array}{c}
\left(\sum_{i=1}^{n}\left|x_{i}\right|^{P}\right)^{\frac{1}{P}}, p \in(1, \infty) ; \\
\max I x I, P=\infty \\
i=1,2, \ldots n
\end{array}\right.
$$

Norm of a vector is calculated by taking $\mathrm{P}^{\text {th }}$ power of each element and adding them. Usually Normed vector space is concerned to discrete and finite domain signals. If $\mathrm{P}=2$ it is energy and $\mathrm{P}<1$ it is called as bounded norm or quasi norm which is highly non convex. It means if we connect two points using straight line then that line will not touches any other point which is defined in vector. 
The result will be a new kind of signal means no other signals exist in between them.
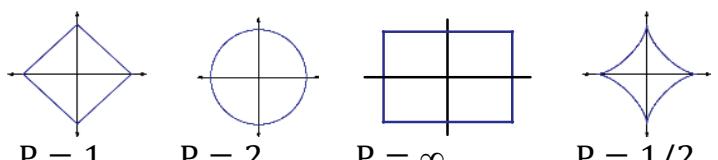

$\mathrm{P}=1 \quad \mathrm{P}=2$

$$
\mathrm{P}=\infty
$$$$
\mathrm{P}=1 / 2
$$

Fig.4. Unit Sets in Two Dimensional (R2) for the $\mathrm{L}_{\mathrm{P}}$ norms

Quasi norm can be defined by equation (6).

$$
\|\mathrm{x}\|_{0}:=|\operatorname{supp}(\mathrm{x})| \text { where } \operatorname{supp}(\mathrm{x})=\left\{\mathrm{i}: \mathrm{x}_{\mathrm{i}} \neq 0\right\}
$$

This quasi norm denotes the coordinates of sparse signal which is having nonzero coefficient. These Lp quasi norm exhibits different properties for different values of. Strength of the signal is measured using above Lp norms. If ' $x$ ' is element of vector defined in two dimensional spaces. Reconstruction of ' $\mathrm{x}_{\mathrm{c}}$ ' in one dimensional affine space is to minimize approximation error $\left(\mathrm{x}-\mathrm{x}_{\mathrm{c}}\right)$ using Lp norms. The choice of will play major role in minimizing the error. Larger value of will increase the error. Bounded norm as shown in Fig. 5 means less than one closely estimate the value. In CS same concept is extended to higher dimensional space to reconstruct the signal.

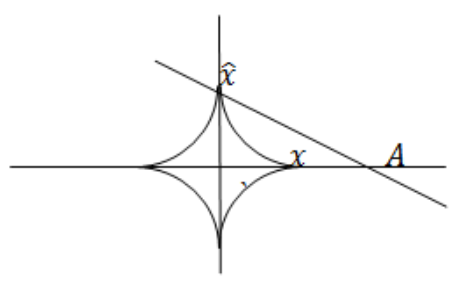

Fig.5. $\mathrm{P}=1 / 2$ Bounded P Norm

\section{COMPRESSIVE SENSING}

\section{A. Sampling Theory}

Actual sampling is operating on analog signal which is essentially a linear identity operator. This operation can be modeled using matrix $Y=\Phi X$ as shown in figure Fig. 6 .

$\mathrm{Y} \Phi=$ I. N X N Identity matrix
N X 1 $\left[\begin{array}{c}\mathrm{y} 1 \\ \mathrm{y} 2 \\ \mathrm{y} 3 \\ \mathrm{y} 4 \\ \vdots \\ \vdots \\ \mathrm{yn}\end{array}\right]\left[\begin{array}{ccccccc}1 & 0 & 0 & 0 & \cdots & \cdots & 0 \\ 0 & 1 & 0 & 0 & \cdots & \cdots & 0 \\ 0 & 0 & 1 & 0 & \cdots & \cdots & 0 \\ 0 & 0 & 0 & 1 & \cdots & \cdots & 0 \\ \vdots & \vdots & \vdots & \vdots & \ddots & \cdots & \vdots \\ \vdots & \vdots & \vdots & \vdots & \vdots & \ddots & \vdots \\ 0 & 0 & 0 & 0 & \cdots & \cdots & 1\end{array}\right] \quad\left[\begin{array}{c}\mathrm{x} 1 \\ \mathrm{x} 2 \\ \mathrm{x} 3 \\ \mathrm{x} 4 \\ \vdots \\ \vdots \\ \mathrm{xn}\end{array}\right] \mathrm{N} \mathrm{X1}$

Fig.6. Sampling Model

' $\mathrm{X}$ ' is a analog signal and ' $\mathrm{Y}$ ' is digital signal which is obtained by multiplying by identity matrix on analog signal. Since operator is linear identity matrix then samples are as per Nyquist rate and $\mathrm{Y}=\mathrm{X}$. Two important points are to be highlighted whenever mathematical model is designed for sampling process. First one is information preservation and second one is reconstruction. Since $\mathrm{Y}=\mathrm{X}$ naturally information is preserved and by using some trivial operation will gives back from.

Sampling, processing without aliasing and reconstruction using SINC interpolation are linear only compression that is converting ' $\mathrm{N}$ ' to ' $\mathrm{K}$ ' is non linear. In compressive sensing we are not interested in all the samples we are interested in sparse samples. Sampling model is modified to incorporate compressive theory.

\section{B. Compressive Theory}

Compressive sensing is all about to cut off the middle concept that is sampling process and develop new type of device which captures the light coming from object and straight away to ' $\mathrm{M}^{\prime}$ measurement [8] which is as same as ' $\mathrm{K}$ ' sparse signal as shown in Fig. 7 . When data is compressible, it can be directly acquire a few representations with little information loss which are negligible.

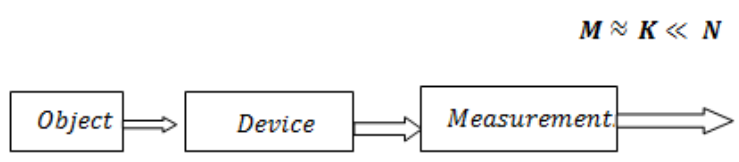

Fig.7. Block Diagram of Compressive Sensing

This dimensionality reduction can also be represented as $\mathrm{Y}=\Phi \mathrm{X}$ where Fil is $\mathrm{MXN}$ which contain information related to sparse measurements as shown Fig. 8 such as co ordinates, coefficient and basis. These sparse signals are obtained by transforming in to new basis and keeping large coefficients and truncating small coefficients. The number of compressive measurements must be close to Fi. It means $\mathrm{M} \approx \mathrm{K} \ll \mathrm{N}$. These measurements are sparse signal. Each measurement carries same amount of information which is called as democratic [10]. In CS mathematical model we are not multiplying by big identity matrix but we are multiplying by short matrix. Present CS is all about discrete and finite system. We are using non adaptive measurement which means they do not depend on previously acquired measurements. CS work starts by assuming measurements are available and by ignoring how to acquire measurements.

$M X 1\left[\begin{array}{c}y 1 \\ y 2 \\ \vdots \\ y m\end{array}\right]=\left[\begin{array}{ccccccc}\Phi_{11} & \Phi_{12} & \Phi_{13} & \Phi_{14} & \cdots & \cdots & \Phi_{1 N} \\ \Phi_{21} & \Phi_{22} & \Phi_{23} & \Phi_{24} & \cdots & \cdots & \Phi_{2 N} \\ \vdots & \vdots & \vdots & \vdots & \vdots & \vdots & \vdots \\ \Phi_{M 1} & \Phi_{M 2} & \Phi_{M 3} & \Phi_{M 4} & \cdots & \cdots & \Phi_{M N}\end{array}\right]\left[\begin{array}{c}x 1 \\ x 2 \\ x 3 \\ x 4 \\ \vdots \\ \vdots \\ x n\end{array}\right] N X 1$

Fig.8. Mathematical Model of CS Measurements

Since we are interested in only sparse measurement therefore sparse matrix is too short so that only sparse samples will be extracted from analog signal ' $\mathrm{X}$ '. Now for 
this CS model we have to verify information preservation. Since ' $\Phi$ ' is too short and it does not have rank ' $n$ ' so information is not preserved. There are infinitely other ' $x$ ' which will give exact same ' $\mathrm{y}^{\prime}$ which means $\mathrm{X}=\Phi^{\mathrm{T}} \mathrm{Y}$ does not give unique solution. Therefore same mathematical model is modified to incorporate information preservation. Since ' $\mathrm{X}$ ' is analog signal which contain ' $\mathrm{K}$ ' sparse signal and in CS importance will be given to sparse signal.

$Y X 1\left[\begin{array}{c}y 1 \\ y 2 \\ \vdots \\ y m\end{array}\right]=\left[\begin{array}{ccccccc}\Phi_{11} & \Phi_{12} & \Phi_{13} & \Phi_{14} & \cdots & \cdots & \Phi_{1 K} \\ \Phi_{21} & \Phi_{22} & \Phi_{23} & \Phi_{24} & \cdots & \cdots & \Phi_{2 K} \\ \vdots & \vdots & \vdots & \vdots & \vdots & \vdots & \vdots \\ \Phi_{M 1} & \Phi_{M 2} & \Phi_{M 3} & \Phi_{M 4} & \cdots & \cdots & \Phi_{M K}\end{array}\right]\left[\begin{array}{c}x 1 \\ x 2 \\ \vdots \\ x k\end{array}\right] K X 1$

Fig.9. Modified Mathematical Model of CS Measurements

First key realization is if we assume ' $\mathrm{X}$ ' is sparse signal then ' $\mathrm{Y}$ ' is a linear combination of ' $\mathrm{K}$ ' columns which are corresponds to the coordinates of ' $\mathrm{X}$ ' . CS model is modified by including $\mathrm{MXK}$ sparse matrix as shown in Fig. 9 . But it is difficult to identify columns of sparse vector since we do not know where the big coefficients are present. In practice it is very complex. If we are able to identify ' $\mathrm{K}$ ' columns then it is a very simple problem. Second key realization is to design better sparse matrix of dimensions $\mathrm{MXK}$ such that it should have rank ' $\mathrm{K}$ ' or select ' $\mathrm{K}$ ' columns which are orthogonal to each other.

\section{DESIGN OF SENSING MATRIX}

This design is based on three important matrix properties

- $\quad$ Null Space Property

- Restricted Isometric property

- Bounded Coherence.

For recovery there should not be two vectors $\mathrm{x}, \mathrm{x}^{\prime}$ such that $\Phi \mathrm{x}=\Phi_{\mathrm{x}}^{\prime}$ because it would be impossible to differentiate among vectors from single measurement. Null space satisfies above requirement since null space means no vectors which are defined by equation (7).

$$
\mathrm{N}(\Phi)=\{z: \Phi z=0\}
$$

Using null space property it is proved that for any $y \in \mathrm{R}^{\mathrm{m}}$ there exist at most one signal $\mathrm{x} \in \Sigma_{\mathrm{k}}$ such that $\mathrm{y}=\Phi \mathrm{x}$ if and only if spark $(\Phi)>2 \mathrm{~K}$. Spark of matrix is defined as smallest number of columns which are linearly independent. Critical design problem of compressive sensing is to design $\Phi$ of dimension $\mathrm{MXN}$ such that if we take arbitrarily columns out then it should have rank and these columns should be orthogonal to each other. This is called Restricted Isometric Property [6], [7]. Structural representation of designed $\Phi$ is as shown in Fig. 10 for $K=3$ and $M=3$. Where sparse matrix contains coefficient values which are taken randomly which means value of ' $i$ ' and ' $j$ ' are any random values.

$$
\begin{gathered}
\text { Arbitrary Columns } \\
M X 1 \quad\left[\begin{array}{l}
y 1 \\
y 2 \\
y 3
\end{array}\right]=\left[\begin{array}{lll}
\Phi_{i j} & \Phi_{i j} & \Phi_{i j} \\
\Phi_{i j} & \Phi_{i j} & \Phi_{i j} \\
\Phi_{i j} & \Phi_{i j} & \Phi_{i j}
\end{array}\right] \quad\left[\begin{array}{l}
x 1 \\
x 2 \\
x 3
\end{array}\right] K X 1
\end{gathered}
$$

Fig.10. Sparse Matrix Obtained Using RIP

Assume that two sparse signal located in ' $\mathrm{K}$ ' dimensional sub space with some Euclidian distance in $\mathrm{R}^{\mathrm{N}}$.
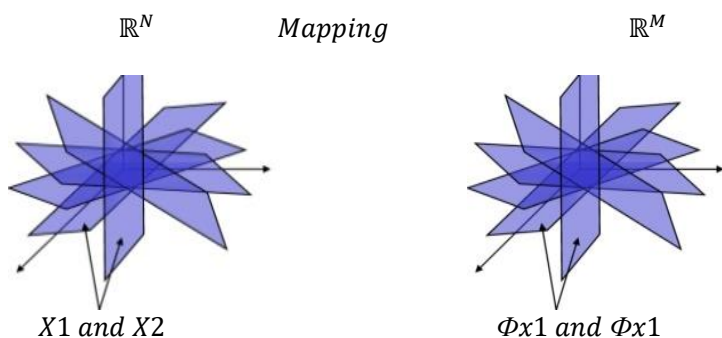

Fig.11. Geometrical Representation of Information Preservation

When it is projected means mapping from $\mathrm{R}^{\mathrm{N}}$ to $\mathrm{R}^{\mathrm{M}}$ (dimensionality reduction) as shown in Fig. 11 using $\Phi$ then above two points will be located at same distance apart. This is nothing but distance preservation and indirectly it is information preservation. RIP ensures that $\|\mathrm{x} 1-\mathrm{x} 2\| 2 \approx\|\Phi \mathrm{x} 1-\Phi \times 2\| 2$ which means information preservation. But it is too complex and too lengthy because we have to repeat taking arbitrarily $F_{i}$ columns from $\mathrm{M} \times \mathrm{N}$ matrix for every possible combinations means $\mathrm{N}^{\mathrm{K}}$ times and out of all selection we have to find every possible ' $\mathrm{K}$ ' column which obeys RIP property. Since 'N' are million (Mega Pixel) dimensions so it is too complex and too lengthy. But some mathematician reduced the complexity by deriving a formula (8) for random matrix which is called as randomized technique [14]. Most of the random matrix (Gaussian matrix, Bernouli etc) have high probability to exhibits RIP property as long as following formula holds

$$
\mathrm{M}=\mathrm{O}\left(\mathrm{Klog}\left(\frac{\mathrm{N}}{\mathrm{K}}\right)\right) \ll \mathrm{N}
$$

Number of measurement grows linearly by ' $K$ ' and logarithmically by ' $N$ '. We can preserve information in signals by acquiring them by randomized sensing. Instead of sampling, linear measurements are taken by random set of weights applied over entire signal and adding them to construct the ' $Y$ ' which is all about information preservation in CS. Coherence (9) is one of the properties of sparse matrix which enables us to recover the signal. It is defined as "largest absolute inner product between any two columns of sparse matrix.

$$
\mu(\Phi)=\max \frac{\left|<\Phi_{i}, \Phi_{j},>\right|}{\left\|\Phi_{i}\right\| 2\left\|\Phi_{j}\right\| 2} \quad \text { for } 1 \leq \mathrm{i}<\mathrm{j}
$$


This coherence will define the range that is $\mu(\Phi) \epsilon$ $\left[\sqrt{\frac{\mathrm{N}-\mathrm{M}}{\mathrm{M}(\mathrm{N}-1)}}, 1\right]$.If $\mathrm{N} \gg \mathrm{M}$ then lower bound becomes $\mu(\Phi) \geq$ $\frac{1}{\sqrt{\mathrm{M}}}$. Since is million dimensions it is difficult to find out all possible columns which are having RIP property. Therefore initially it is mentioned too complex and too lengthy but now because of coherence property complexity is reduced by defining the range. It means signal which is to be recovered is somewhere in that defined range. These techniques are used in Compressive Sensing Based Greedy Pursuit Reconstruction Algorithms [26].

\section{COMPRESSIVE RECONSTRUCTION}

$Y$ M has nul space of dimension ' $\left.-m^{\prime} \quad \begin{array}{c}X \\ y 1 \\ y 2 \\ \vdots \\ y m\end{array}\right]=\left[\begin{array}{ccccccc}\Phi_{11} & \Phi_{12} & \Phi_{13} & \Phi_{14} & \cdots & \cdots & \Phi_{1, n-m} \\ \Phi_{21} & \Phi_{22} & \Phi_{23} & \Phi_{24} & \cdots & \cdots & \Phi_{2, n-m} \\ \vdots & \vdots & \vdots & \vdots & \vdots & \vdots & \vdots \\ \Phi_{m 1} & \Phi_{m 2} & \Phi_{m 3} & \Phi_{m 4} & \cdots & \cdots & \Phi_{m, n-m}\end{array}\right]\left[\begin{array}{c}x 1 \\ x 2 \\ x 3 \\ x 4 \\ \vdots \\ \vdots \\ x n\end{array}\right]$

Fig.12. Given $\mathrm{Y}=$ Fix. For which we have to find out $\mathrm{x}$

Reconstruction means for given ' $\mathrm{Y}$ ' as shown in Fig. 12 (measurements) how to get ' $\mathrm{X}$ '. Random projection does not have full rank but we can exploit the fact that we are not interested in acquiring aerial ' $\mathrm{X}$ ', we are interested in acquiring sparse ' $x$ ' in CS. For this we are using geometrical structure of sparse signal and Normed vector space. Geometrically null space of is represented using hyper plane of dimension $\mathrm{n}-\mathrm{m}$ as shown in Fig. 13 which is aligned with coordinate axis with certain angel. All the 'x's such that $\Phi x$ lives on null space translated to the point ' $x$ '. It means solution is somewhere in the hype plane.

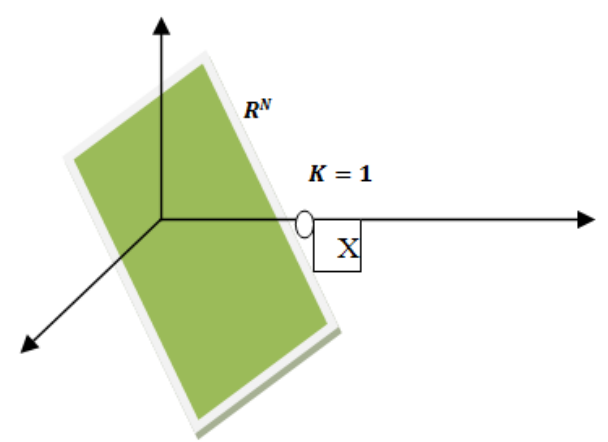

Fig.13. Geometrical structure of $F_{i}$ of n-m dimensions

But on the hyper plane there are infinite $\mathrm{x}^{\prime}$ s present over there. Then search in null space for the best ' $x$ ' according to some criteria like least square. Using $l_{2}$ norm which is defined in equation (11) optimize the problem. Optimization (10) means find out the minimum Normed ' $\mathrm{X}$ ' out of all the ' $\mathrm{x}$ ' which gives $\mathrm{Y}=\Phi \mathrm{x}$.

$$
\text { Optimization } \quad \hat{x}=\arg \min \|x\| 2
$$

Solution using $l_{2} \quad \hat{x}=\left(\Phi^{T} \Phi\right)^{-1} \Phi^{T} Y$

In this we are finding minimum energy signal present in the signal. Geometrical solution using $l_{2}$ norm as shown in Fig.14 is blowing up the sphere which is at origin until it touches the hyper plane at some random angle. The point of touch between sphere and hyper plane is smallest energy signal which agrees above equation. That is the best $\hat{X}$ according to $l_{2}$ optimization.

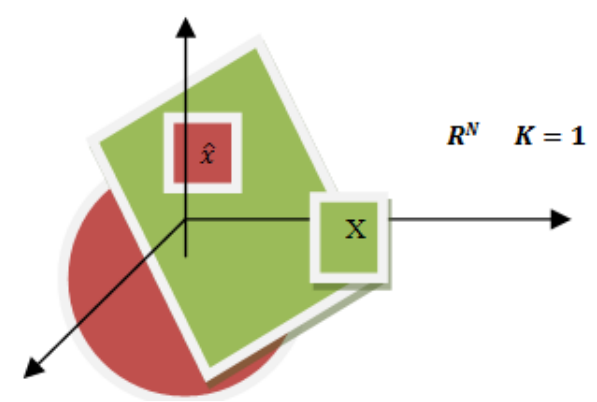

Fig.14. Geometrical Solution Using $l_{2}$

This solution is bad because the sphere and hyper plane touches far away from coordinate axis with random angel so it is not proper signal. Using $\mathrm{l}_{0}$ minimization (12) reconstruction is improved. Graphically In this method instead of searching minimum energy signal ' $x$ ', we have to search sparsest $\mathrm{F}$ in the plane.

$$
\text { Optimization } \quad \hat{x}=\arg \min \|x\| 0
$$

Since $\mathrm{N}$ is million (Mega Pixel) and $\mathrm{K}$ is in terms of 10000. This is solved using NP hard algorithm and these algorithm searches all ' $\mathrm{K}$ ' dimensional subspace to find out sparsest ' $\mathrm{X}$ '. Important factor about these algorithms is that same algorithm can be used to reconstruct the signal irrespective of its basis. Recently some mathematician uses $l_{1}$ minimization (13) for signal reconstruction.

$$
\text { Optimization } \quad \hat{\mathrm{x}}=\arg \min \|\mathrm{x}\| 1
$$

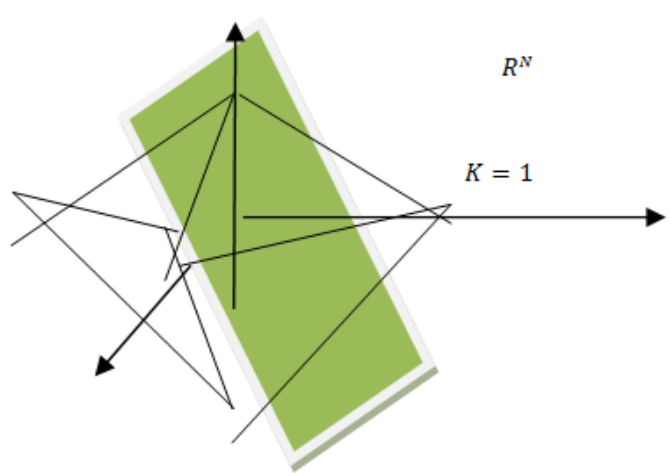

Fig.15. Geometrical Solution Using $1_{0}$ minimization

Here taking the magnitude of the entire coefficient in the vector and add them will give one sparse signal. Like this we have to repeat for all sub spaces and finally original signal is recovered by using polynomial linear algorithm. Geometrically (FIG. 15 ) it is as same as $\mathrm{L}_{2}$ 
optimization only difference is we are using pointy structure instead of sphere. This pointy structure may be diamond. When it is blown it pierces at coordinate axis. That point of intersection is the recovered signal.

\section{PROPOSED WORK AND ITS LITERATURE SURVEY}

In this work we propose optimized high speed VLSI implementation of Compressive Sensing reconstruction algorithm using FPGA as a prototype.

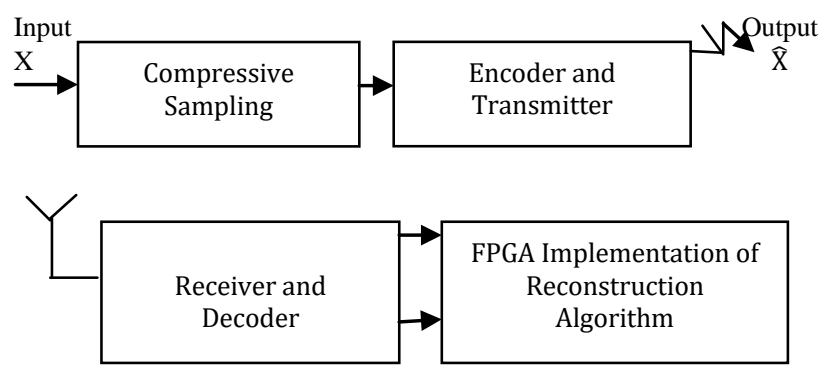

Fig.16. Block Diagram of Proposed Work

Literature Survey: Patrick Machler, Chiristoph, David Bellasi proposed two VLSI architectures in their work [15] titled as "VLSI Design of AMP for Signal Restoration and Compressive Sensing". First architecture referred as AMP-M; second one as AMP-T architecture. Both architectures implements AMP algorithm [16] to reconstruct the compressed signal. One major difference is in sensing matrix. In AMP-M architecture sensing matrix consists of random linear measurement which is build using MAC units, whereas AMP-T consist of aggression of linear measurement which is build using FFT and its inverse FFT units in architecture. These two architectures are mapped on XC6SLX9 FPGA and XC6SLX75 for the problem size $512 \times 1024$ for reconstruction of real time audio signal. Results of both the architectures are compared at the end of the paper. In this paper it is shown that throughput of AMP-M is 4 times greater and AMP-T is 8 times greater than ASIC design. This paper concludes with following future work.

- Theoretical analysis is to be done in presence of fixed point arithmetic.

- $\quad$ To implement AMP on real time Image as well as on Video.

- Early termination

Avi Septimus and Raphel Steinberg published a paper entitled as "Compressive Sampling Hardware Reconstruction". In this paper [17] OMP (Orthogonal Matching Pursuit [18]) used to reconstruct the signal. Compressed signal is mathematically modeled as $\mathrm{y}=\Phi \mathrm{x}$, where $x$ is $i / p$ signal, $y$ is number samples and $\Phi$ is sensing matrix. Minimum number of samples required to reconstruct is represented with the formula $\mathrm{O}(\mathrm{m} \cdot \log \mathrm{N})$. This algorithm mainly consists of two stages. In the first stage number of sparse $(\mathrm{m})$ is identified where as in second stage value of reconstructed signal is calculated using some iteration as per the algorithm. Using VHDL code matrix inverse unit is designed which is required to implement one of the concept used in OMP. This block is incorporated in OMP unit. This unit will take y and $\Phi$ as a input to reconstruct signal $\mathrm{x}$. Results of OMP algorithm is compared with GPU implementation which is done on other processor, which indicates that OMP hardware implementation require almost negligible time. This design is synthesized on Xilinux Vertex 5 FPGA for the problem size $32 \times 128$ with $\mathrm{m}=5$. Paper concluded with following future work.

- Need to develop consumer product using OMP unit.

- To replace existing ADC in real time application.

Guoyan Li, Junhua and Qingzeng Song [19] published paper entitled as "The Hardware Design and Implementation of Signal Reconstruction Algorithm Based on CS". They used CG (Conjugate Gradient) algorithm [20]. Both AMP \& OMP method reconstruction has a high precision, large complex calculations and speed is very less. To improve above parameter parallel and pipelined FPGA mapping is proposed. CG algorithm stars at interior part of rising or falling edge of the signal and later proceeds with downwards. This iterative method is called as inferior point method. CG algorithm contains some calculations based on multiplication \& division on vectors. Multiplication part is build using FPGA's MAC unit, whereas division is implemented through software. Both parts are combined and it is called as CG processor. This processor implements internal point method whereas remaining part of the algorithm is implemented in other hardware. These two hardware unit is coupled with control module and they work independently which is the inherent property of $\mathrm{CG}$ algorithm. This works on matrix size of $24 \times 24$, which is build using 8 MAC unit as well as reuse concept. Entire design is mapped on Altera Cyclone II - EP2C70F896C6 FPGA. Software experiment is implemented DSP Builder 11.1 tools. Paper is concluded with following future work

- $\quad$ Need to develop more \& more parallel unit to improve complexity.

Patric Machler, Lin Bai, Hubert and Michel published paper [21] as "High Speed Compressed Sensing Reconstruction on FPGA Using OMP and AMP". Compressed sensing is mathematically modeled as $\mathrm{y}=\Phi \mathrm{x}$, where $\mathrm{x}$ is $\mathrm{i} / \mathrm{p}$ signal, $\Phi$ is non-adaptive linear projections. Recovering original signal is done by solving $\mathrm{y}=\Phi \mathrm{x}=$ $\mathrm{y}=\Phi \psi \mathrm{s},(\psi \mathrm{s}$ is wavelet transform), under certain conditions OMP uses the concept of adding best fitting element in each steps of algorithm. AMP uses thresholding concept to refine the estimation in step as per the algorithm. OMP consist of matrix multiplication as well as least square optimization operations. To reduce the complexity of operations, large number of parallel multiplier is instantiated using MAC units and it configured as vector multiplication unit (VMU). For this VMU parallel data (Fixed floating point arithmetic) is supplied through 
sequential circuits such as RAM, ROM, REG, SRR and MAX. AMP uses modified version of VMU, which is operated in two modes that is parallel and serial mode. Both algorithm uses problem size of $256 \times 1024$. These are implemented on Xilinux vertex-6 (XC6VLX240T). FPGA is tested in PC through UART interface by taking Lena image of size $256 \times 256$ pixels as a test input. OMP achieved $23.5 \mathrm{db}$ signal to noise ratio, whereas AMP 21.4db. These FPGA results are compared with MATLAB reconstruction, which states that OMP is 4000 \& AMP is 5000 times faster than software.

Pierre B, Hassan R and Abbes A, published a paper [22] named as "High level Prototyping FPGA implementation of the OMP". Authors modified the OMP algorithm by comparing previous implementation by Aseptimus and $\mathrm{R}$ Steinberg [17]. The main theme of the paper is to reduce execution time and FPGA area. In this architecture three main critical part of the algorithm is identified as Vector Multiplication, least square problem and estimation of the signal. These problems are tackled by improving hardware which are used in previous case [17]. Complex operations are implemented on MAC units. A general control unit is designed to sequence the operations as per the algorithm. The problem definition is as same as previous one i.e $32 \times 128$ with $m=5$. This algorithm is synthesized on ML506 board which contains Vertex 5 FPGA (XC5VSX50T). The result is initially compared with software (MATLAB), which states that software requires $606 \mu$ s where as hardware $16 \mu$ s execution time. Later result is compared with existing which requires $24 \mu \mathrm{s}$ which is not a significant change. Area is reduced using reusable concept of FPGA. At the end it is concluded with fallowing future work

- Need to develop more parallel and pipelined structure with different problem size

Jerome $\mathrm{S}$ and Tinoosh $\mathrm{M}$ improved the performance of OMP in their work [23] published as "High performance CS reconstruction Hardware with QRD [24] process". They took previous paper [25] as a reference. Compressed signal is modeled as $\mathrm{y}=\Phi \mathrm{x} \& \Phi$ is a DxN measurement matrix. They identified critical problem as dot product calculation (Optimization) and division operation (Least square) of the algorithm in previous paper. Optimization problem is solved by finding ' $\mathrm{m}$ ' indices of $\Phi$ with different architecture. Least square problem is tackled by QRD process instead of Cholesky Decomposition method. The problem size is $64 \times 256$ with $\mathrm{m}=8$ is implemented in 65nm CMOS technology with Verilog code, RTL compiler and Cadence software. Results indicate that execution time is $13.7 \mu \mathrm{s}$ with chip area $0.69 \mathrm{~mm} 2$. Later same problem is implemented on Xilinux Vertex 5 FPGA, which takes execution time $27.14 \mu$ s. When problem size reduced to $64 \times 128$ with $m=5$, then execution time is $10 \mu \mathrm{s}$. Finally paper is concluded with future work mentioned bellow.

- To avoid trade of between execution time and problem size.
In 2013 again Jerome $\mathrm{S}$ and Tinoosh $\mathrm{M}$, updated their previous work [23] with less complexity in a paper [25] "Low Complexity FPGA Implementation of CS Reconstruction". In this paper they concentrated on same Optimization and Least square problem with QRD method. A new thresholding method is used in QRD process. This eliminates certain columns of $\Phi$ using thresholding concept which is taken as $\alpha$. This $\alpha$ is updated at the end of each iteration. Mean Square error of estimated signal increases with $\alpha$ Improved OMP is synthesized on Xilinux Vertex 5 (XC5VL110T). Results are verified for the various $\alpha$ value $(0.05$ to 0.5$)$ of problem size $64 \times 256$ with $m=8$. It is observed that reconstruction time decreases with $\alpha$. Results are compared with Septimus [17] work, which indicates that execution time is 3.4 times faster. When it is implemented with $64 \times 128$ with $\mathrm{m}=5$ and $\alpha=0.25$, it will take $7.13 \mu \mathrm{s}$, whereas previous work [15] will take $10 \mu$ s execution time. Future work is

- Selecting proper value of $\alpha$ for different dimension if QRD thresholding concept is used in OMP algorithm.

\section{CONCLUSION AND FUTURE WORK}

Compressive sensing is one of the emerging fields which may used as alternative method for Sampling Theorem for all the applications. This field is not yet commercialized means still lot of concrete work is required in various discipline like statistics, coding, electronics, computer science etc. Some overall future works are required which is listed below.

- Need to design new kind of electronic device which converts analog signal into measurements.

- Need to design Sparse Matrix which obeys NULL SPACE, RIP and COHERENCE property.

- To develop ADC type of universal devices such that it can be replaced in the existing products with negligible cost.

- Need to develop algorithm or some software/hardware which gives the output straight away into sparse matrix.

- Need to develop more Greedy and Polynomial algorithm for reconstruction.

- Need to extend CS theory for video and live streaming.

This paper highlights theoretical part of Compressive Sensing which may create interest to readers to work on this field. Some of the mathematical part is explained using graphical method to create interest. Finally Compressed Sensing will become one of the common tools for majority of the applications in future.

\section{REFERENCES}

[1] David L. Donoho, "Compressed Sensing," IEEE Trans. Information Theory, vol. 52, no. 4, pp.1289-1306, Apr. 2006. 
[2] Emmanuel J. Candes and Michel B.Wakin "Compressive Sampling" IEEE signal processing Magazine 21 March 2008.

[3] Richard Baraniuk, "Lecture Notes: Compressive Sensing," IEEE Signal Processing Magazine, p.118-121, July, 2007.

[4] Justin Romberg, "Imaging via compressive sampling," IEEE Signal Processing Magazine, 25(2), pp. 14 - 20, March 2008.

[5] R. Baraniuk. Compressive sensing. IEEE Signal Processing Mag., 24(4):1188211; 120, 124, 2007.

[6] R. Baraniuk, M. Davenport, R. DeVore, and M. Wakin. "A simple proof of the restricted isometric property for random matrices". Const. Approx., 28(3):2538211; 263, 2008.

[7] E. Cand [U+FFFD] "The restricted isometric property and its implications for compressed sensing". Comptesrendus de l'Acad [U+FFFD]e des Sciences, S[U+FFFD]e I, 346(9-10):5898211;592, 2008.

[8] M. Davenport, P. Boufounos, M. Wakin, and R. Baraniuk. "Signal processing with compressive measurements". IEEE J. Select. Top. Signal Processing, 4(2):4458211; 460, 2010.

[9] M. Davenport, J. Laska, P. Boufouons, and R. Baraniuk. "A simple proof that random matrices are democratic". Technical report TREE 0906, Rice Univ., ECE Dept., Nov. 2009.

[10] R. DeVore. Deterministic constructions of compressed sensing matrices. J. Complex., 23(4): 9188211; 925, 2007.

[11] M. Duarte and R. Baraniuk. Kronecker "compressive sensing". Preprint, 2009.

[12] A. Gilbert and P. Indyk. "Sparse recovery using sparse matrices." Proc. IEEE, 98(6):9378211; 947, 2010.

[13] S. Muthukrishnan. "Data Streams: Algorithms and Applications", volume 1 of Found. Trends in Theoretical Comput. Science. Now Publishers, Boston, MA, 2005.

[14] Richard Baraniuk, Mark Davenport, Ronald DeVore, Michael Wakin "A Simple Proof of the Restricted Isometric Property for Random Matrices". Published online: 15 January 2008 (C) Springer Science+Business Media, LLC 2008.

[15] P. Maechler, C. Studer, D. Bellasi, A. Maleki, A. Burg, N. Felber, H. Kaeslin, and R. Baraniuk, "VLSI design of approximate message passing for signal restoration and compressive sensing," IEEE Journal on Emerging and Selected Topics in Circuits and Systems, vol. 2, no. 3, 2012.

[16] D. Donoho, A. Maleki, and A. Montanari, "Messagepassing algorithms for compressed sensing," in Proc. of the National Academy of Sciences, vol. 106, no. 45, Sep. 2009, pp. 18 914-18919.

[17] A. Septimus and R. Steinberg, "Compressive sampling hardware reconstruction," in Circuits and Systems (ISCAS), Proceedings of 2010 IEEE International Symposium on, 2010, pp. 3316-3319.

[18] J. A. Tropp, Anna and C. Gilbert, «Signal recovery from random measurements via Orthogonal Matching Pursuit,» IEEE Trans. Inform. Theory, vol. 53, pp. 4655-4666, 2007.

[19] Guoyan Li, Junhua and Qingzwng Song, "The Hardware design and implementation of a signal reconstruction algorithm based on compressed sensing", in Intelligent Networks and Intelligent Systems, Proceedings of 5th IEEE International Symposium on, 2012.

[20] Antonio, Roldao, Lopes and George "A Constantinides. A Throughput FPGA-Based Floating Point Conjugate Gradient Implementation". Lecture Notes in Computer Science, 2008, Volume 4943, pp.75-86.
[21] Lin Bai, Patrick Maechler, Michel and Hubert Kaeslin, "High Speed Compressed Sensing Reconstruction on FPGA using OMP and AMP”, IEEE 2012.

[22] Pierre B, Hasan R and Abbes A, "High Level Prototyping and FPGA Implementation of The OMP Algorithm", in Information Sciences, Signal Processing and their Application Conference, 11th IEEE International Symposium on, 2012.

[23] J. Stanislaus and T. Mohsenin, "High performance compressive sensing reconstruction hardware with qrd process," in Circuits and Systems (ISCAS), 2012 IEEE International Symposium on, may 2012, pp. 29-32.

[24] O A. Irturk, S. Mirzaei, and R. Kastner, "An Efficient FPGA Implementation of Scalable Matrix Inversion Core using QR Decomposition," 2009. [Online]. Available: http://csetechrep.ucsd.edu/Dienst/UI/2.0/Describe/ncstrl.uc sd_cs e/CS2009-0938.

[25] J. Stanislaus and T. Mohsenin, "Low-complexity fpga implementation of compressive sensing reconstruction, "International Conference on Computing, Networking and Communications, Multimedia Computing and Communications Symposium, August 2013.

[26] Meenakshi and Sumit Budhiraja, "A survey of Compressive Sensing Based Greedy Pursuit Reconstruction Algorithms", IJIGSP, vol. 7, No.10, PP.110, Pub. Date: 2015-9-8.

\section{Authors' Profiles}

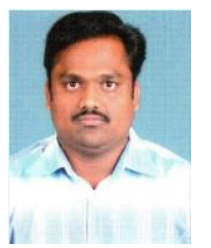

Santosh S. Bujari received his master's degree in Digital Electronics from Visvesvaraya Technological University, Belgum, India in 2005. Since 2005 he has been working as faculty in SKSVMA College of engineering and technology, Laxmeshwar, India. Currently he is pursuing a Ph.D at VTU Belgaum, India. His research interest includes VLSI and Compressive Sensing.

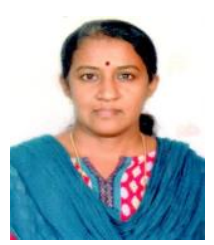

Saroja V Siddamal, received the B.Tech. degree in Electronics and Communication Engineering, and M.E in Digital Electronics from Karnataka University, Dharwad in 1995 and 1997. She received Ph.D degree from Department of Electronics and Communication, the Jawaharlal Nehru Technology University at India in 2013. Presently she is serving as Associate Professor in Department of Electronics and Communication at B. V. Bhoomaraddi College of Engineering and Technology, Hubli. Her research interests include VLSI Signal Processing which includes Architectural design and BIST Testing. She advises doctoral students at Vishweshwaraya Technological University, Belgaum and Bangalore. She is faculty advisor for E-Baja 2015-2016, ESVC 2014-2016, HVVC -2015-2016 events. She has authored or co-authored over 25 technical papers in archival journals and refereed international conferences. She is the reviewer of many international conferences. She received best poster award in 2015 International Conference on Transformations in Engineering Education. She is life member of IETE and ISTE. 